\title{
Immunotactoid glomerulopathy associated with mycosis fungoides
}

\author{
A. Torrelo, M.T. Rivera, F. Mampaso, A. España, R. Marcèn, J. Ortuño and \\ A. Ledo
}

Hospital Ramòn y Cajal, Apartado 37, 28034-Madrid, Spain.

\begin{abstract}
Summary: A patient with mycosis fungoides developed a nephrotic syndrome. Renal biopsy revealed deposits of a highly organized fibrillar material which did not stain with the typical amyloid stains; this picture was consistent with the diagnosis of non-amyloidotic fibrillary glomerulopathy or immunotactoid glomerulopathy. We believe this is the first case reported of immunotactoid glomerulopathy associated with mycosis fungoides. Possible pathogenetic implications are discussed with reference to previous publications.
\end{abstract}

\section{Introduction}

In 1977, Rosenmann and Eliakim ${ }^{1}$ described a patient with a nephrotic syndrome associated with deposits of an amyloid-like material in the glomeruli, which consisted of highly organized fibrillar structures composed of immunoglobulins or immune complexes. This material did not stain with typical stains for amyloid (Congo red and thioflavin). The terms non-amyloidotic fibrillary glomerulopathy or immunotactoid glomerulopathy were later accepted in order to differentiate this entity from other lesions associated with glomerular fibrillar deposits such as amyloidosis, cryoglobulinaemia, monoclonal gammopathy, light chain glomerulopathy and systemic lupus erythematosus. This new clinico-pathological syndrome is characterized clinically by proteinuria $(100 \%)$ sometimes in the nephrotic range $(70 \%)$, microscopic haematuria $(70 \%)$, chronic renal insufficiency $(50 \%)$, and acute renal failure $(20 \%){ }^{2}$ Diagnosis is made by finding microtubules $10-20 \mathrm{~nm}$ in diameter located in mesangium and glomerular basement membrane, which do not stain with amyloid stains. High blood pressure, nephrotic syndrome, male sex, and extensive fibrillary deposits are associated with poor prognosis, with progressive deterioration in renal function, leading to end-stage renal failure. ${ }^{3}$

There is only one previous published case of immunotactoid glomerulopathy (IG) associated with a lymphoproliferative disease (chronic lymphocytic leukaemia), ${ }^{3}$ although similar microfibrils

Correspondence: Antonio Torrelo, M.D., Fermin Caballero, 56, 28034-Madrid, Spain

Accepted: 20 April 1990 have been described in a patient with membranous nephropathy and lymphoma. ${ }^{4}$ Thus, we believe this is the first case of immunotactoid glomerulopathy in a patient with mycosis fungoides (MF).

\section{Case report}

A 49 year old white female was admitted to our hospital for re-staging and treatment of MF stage IB (T2N0M0B0). Her initial lesions had first appeared 5 years earlier. She had a history of mild hypertension which was controlled by thiazides, and intermittent oedema in lower limbs. A nonquantified proteinuria was present at her last admission 5 months before. The patient had been treated with PUVA-therapy 4 months before admission. On clinical examination blood pressure was $160 / 100 \mathrm{mmHg}$. She had poikilodermatous lesions in her back and proximal limbs, and erythematous and browny, finely scaling, mildly infiltrated plaques which affected her trunk and limbs. Two lymph nodes were palpable in her right axilla and one in the left; no other enlarged lymph nodes were present. Clinical examination was otherwise unremarkable.

Investigations revealed serum creatinine $124 \mu \mathrm{mol} / 1$ (normal $26.5-106$ ), urea $27.7 \mathrm{mmol} / 1$ (3.05-16.8), creatinine clearance $69.9 \mathrm{ml} / \mathrm{min}$ $(82-142)$, triglycerides $4.19 \mathrm{mmol} / \mathrm{l}(0.33-1.70)$, cholesterol $8.34 \mathrm{mmol} / \mathrm{l}(3.62-6.72)$, and total serum proteins $53 \mathrm{~g} / 1(60-80)$. Urinalysis showed proteinuria in a nephrotic range $(80 \mathrm{mg} / \mathrm{kg} /$ day; weight $55 \mathrm{~kg}$ ), 12-15 red blood cells per field (Addis 23750 erythrocytes per minute), and hyaline, granular and fatty casts. A serum electro- 
phoresis showed albumin $49.7 \%(53-62)$. IgG was $5.9 \mathrm{~g} / 1$ (6.4-13.5). ANA were positive at low titre $(1 / 20)$. Other studies were normal or negative: blood cell count, clotting, IgA and IgM levels, complement studies, cryoglobulins, intradermal tests with PPD and candidine, urine culture, urine electrophoresis, and serum and urine immunoelectrophoresis. Circulating Sèzary cells were not present. Renal ultrasonography showed both kidneys of normal size and morphology. Chest and abdomen roentgenograms and abdominal tomographies were normal.

A skin biopsy specimen obtained from the trunk showed a diffuse lymphohistiocytic infiltrate, including atypical lymphocytes with hyperchromatic, irregularly-shaped nuclei which eventually invaded epidermis. Immunophenotype of the atypical cells was that of mature $T$ helper cells. A lymph node biopsy from the right axilla showed dermatopathic changes; infiltration of atypical cells was not evident. A bone marrow biopsy revealed non-specific reactive changes. A renal biopsy specimen showed by light microscopy a variable increase in the mesangial matrix and the presence of a material deposited in the mesangial area showing a brown colour staining with silver methenamine stain and was negative after Congo red and $T$ thioflavin staining. By direct immunofluorescence, peripheral capillary wall deposits of IgG and C3 were demonstrated. Ultrastructural studies revealed the presence of coarse fibrils with a diameter ranging from 10 to $20 \mathrm{~nm}$, located in the mesangium and glomerular basement membranes (Figures 1 and 2).

Treatment with topical mechlorethamine $(10 \mathrm{mg}$ in $50 \mathrm{ml}$ of water; corporal application once a day) and nifedipine $30 \mathrm{mg} /$ day was started. Ten months after discharge, skin lesions are quiescent and renal function is stable.

\section{Discussion}

Mycosis fungoides is a malignancy of $\mathrm{T}$ lymphocytes which initially involves skin, with eventual dissemination to lymph nodes and internal organs. Our patient developed an immunotactoid glomerulopathy in the context of a MF stage IIA (T2N1M0) without haematic dissemination. Other causes of fibrillary glomerulopathy were excluded.

Invasion of the kidneys by atypical cells in MF is common, ${ }^{5}$ but primary glomerulonephritis in patients with MF has only been described in three cases with advanced disease. ${ }^{6.7}$ In one patient, ${ }^{7}$ renal invasion by atypical cells coexisted with glomerulopathy.

Very little is known about the pathogenesis of immunotactoid glomerulopathy. ${ }^{3}$ An abnormal production of monoclonal immunoglobulins, even

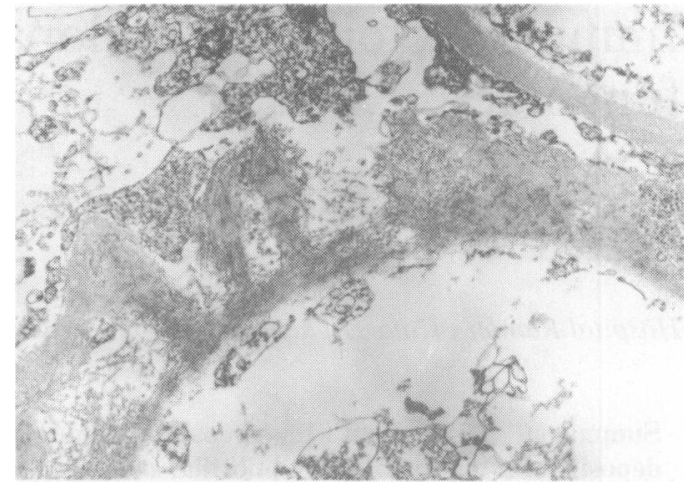

Figure 1 Microfibrillar mesangial deposits sized $10-20 \mathrm{~nm}(\times 5586)$.

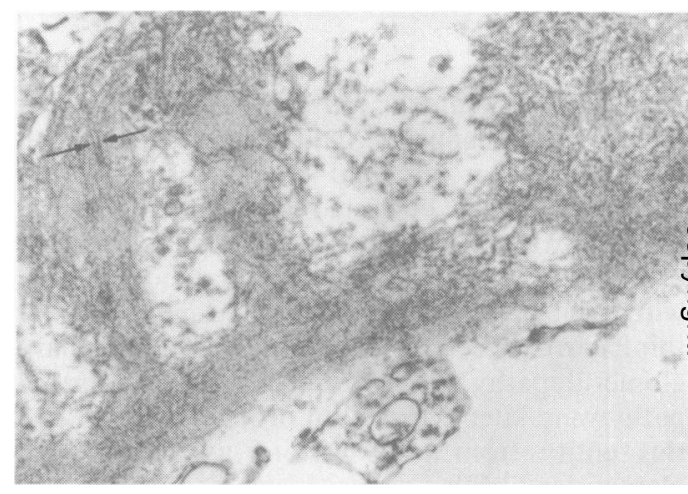

Figure 2 A detail showing immunotactoid microtubules within electrondense deposits (arrows) $(\times 16758)$.

in the absence of a clinical paraproteinaemia or plasmocytosis, or immune complexes of uniform structure could account for the deposits. It is not known why these deposits only appear in renal glomeruli. $^{8}$

Atypical cells in MF usually show immunophenotype of mature helper/inducer $\mathrm{T}$ lymphocytes, ${ }^{9}$ capable of promoting B lymphocyte response, leading to formation of diverse types of autoantibodies and immunoglobulins (mono- or polyclonal). It is well known that MF can coexist with monoclonal gammopathy or multiple myeloma. ${ }^{10}$ In our patient there was no hypergammaglobulinaemia, but diminished IgG, as may be seen in nephrotic syndrome. Likewise, none of the cases of primary immunotactoid glomerulopathy previously described showed elevated levels of immunoglobulins.

A case of immunotactoid glomerulopathy in a patient with a chronic lymphatic leukaemia has 
been reported, ${ }^{3}$ and it has also been reported in association with diseases of the immune system. ${ }^{2,8,11,12}$ In our patient, the appearance of immunotactoid glomerulopathy at an early stage of MF, when most of the atypical cells preserve a T

\section{References}

1. Rosenmann, E. \& Eliakim, M. Nephrotic syndrome associated with amyloid-like glomerular deposits. Nephron 1977, 18: 301-308.

2. Sturgill, B.C. \& Kline Bolton, W. Non-amyloidotic fibrillary glomerulopathy. Kidney Int 1989, 35: 233.

3. Korbet, S.M., Schwartz, M.M., Rosenberg, B.F., Sibley, R.K. \& Lewis, E.J. Immunotactoid glomerulopathy. Medicine 1985, 64: 228-243.

4. Rosenmann, E., Brisson, M.-L., Bercovitch, D.D. \& Rosenberg, A. Atypical membranous glomerulonephritis with fibrillar subepithelial deposits in a patient with malignant lymphoma. Nephron 1988, 48: 226-230.

5. Levi, J.A. \& Wiernik, P.H. Management of mycosis fungoides: current status and future prospects. Medicine 1975,54 73-88.

6. Ramirez, G., Stinson, J.B., Zawada, E.T. \& Moatamed, F IgA nephritis associated with mycosis fungoides. Report of two cases. Arch Intern Med 1981, 141: 1287-1291.

7. Allon, M., Campbell, W.G., Nasr, S.A., Bourke, E., Stoute, J. \& Guntupalli, J. Minimal change glomerulopathy and interstitial infiltration with mycosis fungoides. Am J Med 1988, 84: $756-759$. helper/inducer immunophenotype with possible functional expression supports the hypothesis that immunotactoid glomerulopathy could be a secondary feature of MF.

8. Schifferli, J.A., Merot, Y., Cruchaud, A. \& Chatelanat, F. Immunotactoid glomerulopathy with leucocytoclastic skin vasculitis and hypocomplementemia: a case report. Clin Nephrol 1987, 27: 151-155.

9. Edelson, R.L. Cutaneous T cell lymphoma: mycosis fungoides, Sèzary syndrome, and other variants. J Am Acad Dermatol 1980, 2: 89-106.

10. Venencie, P.Y., Winkelmann, R.K., Friedman, S.J., Kyle, R.A. \& Puissant, A. Monoclonal gammopathy and mycosis fungoides. Report of four cases and review of the literature. $J$ Am Acad Dermatol, 1984, 11: 576-579.

11. Sturgill, B.C., Bolton, W.K. \& Griffith, K.M. Congo rednegative amyloidosis-like glomerulopathy. Hum Pathol 1985 16: 220-224.

12. Kobayashi, Y., Fujii, K., Kurokawa, A., Kamiyama, M. \& Miyazawa, H. Glomerulonephropathy with amyloid-stainnegative microfibrillar glomerular deposits. Nephron 1988, 48: 33-39. 